\title{
Investigación
}

\section{Desigualdades en mortalidad relacionadas con características socioeconómicas en el departamento del Huila, Colombia, 2009-2013}

\author{
Inequalities in mortality related to socioeconomic characteristics in \\ the department of Huila, Colombia 2009-2013
}

\author{
Desigualdades na mortalidade relacionadas a características \\ socioeconômicas no departamento de Huila, Colômbia 2009-2013
}

Carlos A. Montalvo-Arce, ${ }^{1}$ Carlos A. Carmona-Patiño, ${ }^{2}$ Dora Cardona-Rivas ${ }^{3}$

Maestría en Salud Pública. Medicina. Universidad Surcolombiana. Colombia. Correo electrónico: carlos.montalvo@usco.edu.co. ORCID: https://orcid.org/0000-0003-0309-7859

Maestría en Salud Pública, Bacteriología. Secretaría de Salud Departamental del Huila. Colombia. Correo electrónico: cacarmona80@ hotmail.com. ORCID: https://orcid.org/0000-0002-0394-6542

Doctorado en Ciencias Sociales, Bacteriología y Laboratorio Clínico. Universidad Autónoma de Manizales. Colombia. Correo electrónico: dcrivas@autonoma.edu.co.ORCID: https://orcid.org/0000-0002-5378-3518

Recibido: 30/11/2016. Aprobado: 18/07/2017. Publicado: 10/08/2017.

Montalvo-Arce CA, Carmona-Patiño CA, Cardona-Rivas D. Desigualdades en mortalidad relacionadas con características socioeconómicas en el departamento del Huila, Colombia 2009-2013. Rev. Fac. Nac. Salud Pública, 2017; 35(3): 343-357. DOI: 10.17533/udea.rfnsp.v35n3a05

\section{Resumen}

Objetivo: determinar las desigualdades en la mortalidad por causas específicas relacionadas con características socioeconómicas, en municipios del Huila 2009-2013. Métodos: estudio ecológico que comparó las mortalidades por Infección Respiratoria Aguda, cáncer de estómago, enfermedades hipertensivas y cerebrovasculares, accidentes de tránsito, homicidio, diabetes y mortalidad en niñez, con base en su situación socioeconómica, según las variables socioeconómicas Cobertura en Educación Media, NBI, Ruralidad, Suficiencia Económica, Valor Agregado Municipal y el Índice de Calidad del Agua en los 37 municipios del Huila. Se calcularon tasas ajustadas por edad con el método directo y medición de desigualdades confrontando las variables descritas, usando medidas basadas en rango, regresión y desproporcionalidad, usando el software Epidat 4.0. Resultados: en la mortalidad por cáncer gástrico, por enfermedades hipertensivas y en la mortalidad en niñez se identificó constante desigualdad frente a las variables socioeconómicas evaluadas, en contra de los municipios menos favorecidos. En la mortalidad por infecciones respiratorias agudas y homicidios, se observaron mayores tasas en los municipios con mejores condiciones socioeconómicas. Conclusiones: existe gran variabilidad entre las condiciones socioeconómicas de los municipios y entre las mortalidades por los eventos evaluados, observando aceptable correlación entre las diferentes medidas de desigualdades utilizadas.

-Palabras clave: indicadores sociales, enfermedades respiratorias, cáncer de estómago, hipertensión, trastornos cerebrovasculares, diabetes mellitus, accidentes de tránsito, homicidio, mortalidad en la niñez, desigualdades en la salud. 


\begin{abstract}
Objective: to determine the inequalities in mortality due to specific causes related to socioeconomic characteristics in the municipalities of the Huila department, Colombia between 2009 and 2013. Methodology: an ecological study comparing mortality rates due to acute respiratory infection, stomach cancer, hypertensive and cerebrovascular disease, traffic accidents, homicide, diabetes and childhood mortality based on socioeconomic status according to the following socioeconomic variables: secondary education coverage, unsatisfied basic needs, rurality, economic sufficiency, municipality's added value and water quality index. The study included the 37 municipalities of the Huila department. Age-adjusted rates were calculated using the direct method and the inequalities were measured by comparing against the described variables using rank, regression and disproportionality based measures. The Epidat 4.0 software
\end{abstract}

was used for this purpose. Results: child mortality and mortality due to gastric cancer and hypertensive disease showed constant inequality regarding the assessed socioeconomic variables in the less favored municipalities. As for mortality due to acute respiratory infections and homicides, higher rates were observed in municipalities with better socioeconomic conditions. Conclusions: there is great variability in the socioeconomic conditions of the municipalities and the mortality rates due to the assessed events. The authors observed an acceptable correlation between the different measures of inequality used in the study.

-Keywords: social indicators, respiratory diseases, stomach cancer, hypertension, cerebrovascular diseases, diabetes mellitus, traffic accidents, homicide, infant mortality, inequalities in health.

\section{Resumo}

Objetivo: determinar as desigualdades na mortalidade por causas específicas relacionadas a características socioeconômicas nos municípios de Huila entre os anos de 2009 e 2013. Metodologia: estudo ecológico que comparou as mortalidades por infecção respiratória aguda, câncer de estômago, doenças hipertensivas e acidentes vasculares cerebrais, acidentes de trânsito, homicídio, diabetes e mortalidade na infância com base na situação socioeconômica segundo as variáveis socioeconômicas cobertura do ensino médio, NBI, Ruralidade, Suficiência Econômica; Valor Agregado Municipal e o Índice de Qualidade da Água nos 37 municípios de Huila. Foram calculadas as taxas ajustadas por idade com o método direto e a medição de desigualdades, confrontando-as com as variáveis descritas, a partir da utilização de medidas baseadas no intervalo, em regressão e em desproporcionalidade, utilizando-se o software
Epidat 4.0. Resultados: na mortalidade por câncer gástrico, por doenças hipertensivas e na mortalidade na infância foram identificadas constantes desigualdades em relação às variáveis socioeconômicas avaliadas contrárias aos municípios menos favorecidos. Para a mortalidade por infecções respiratórias agudas e homicídios, foram observadas maiores taxas nos municípios com melhores condições socioeconômicas.

Conclusões: existe uma grande variedade entre as condições socioeconômicas dos municípios e entre as mortalidades para os eventos avaliados, observando-se uma correlação aceitável entre as diferentes medidas de desigualdade utilizadas.

-Palavras-chave: Indicadores sociais, Doenças respiratórias, câncer de estômago, hipertensão, transtornos vasculares cerebrais, diabetes mellitus, acidentes de trânsito, homicídio, mortalidade na infância, desigualdades na saúde.

\section{Introducción}

Está actualmente documentada, de forma amplia, la relación existente entre las condiciones en que la gente nace, vive, trabaja y envejece y la situación de salud de las poblaciones [1]. Estas condiciones se relacionan con aspectos sociales, económicos, políticos, culturales y medioambientales, llamados en conjunto Determinantes sociales de la Salud (DSS) [2].

Estas condiciones han establecido estratificaciones en la sociedad (determinantes estructurales), generadoras de desigualdades por sí mismas, entendiendo el término desigualdad como una diferencia entre uno o más grupos comparados. Sin embargo, estas condiciones de desigualdad pueden afectar en forma adversa a las poblaciones más vulnerables y se constituyen en condiciones determinantes para la situación de salud [3]. En consecuencia, las inequidades plantean un juicio moral a las desigualdades y se entienden como diferencias innaturales, injustas, arbitrarias, innecesarias y evitables, determinadas social e históricamente [4].

Con base en el Modelo Conceptual de Determinantes Sociales de la Salud del equipo de equidad de la OMS [5], se comprende la dinámica de generación de desigualdades e inequidades en salud, en un contexto social y político en donde se generan estratificaciones socioeconómicas en ingreso y poder, dando lugar a la identificación de determinantes estructurales $\mathrm{y}$ determinantes intermedios de la salud. Los primeros son aquellos que refuerzan o generan la estratificación social en la sociedad, y definen la posición socioeconómica individual; los segundos son las condiciones materiales de vida, entre ellas las condiciones de trabajo y de 
vivienda, características psicosociales y factores de comportamiento. Los determinantes estructurales operan a través de los determinantes intermedios. Es así como el abordaje de los DSS se convierte en una premisa para el mejoramiento de las condiciones de salud y de vida de las poblaciones, a través de planteamientos reiterativos por parte de la OMS y afirmado en la $62^{\mathrm{a}}$ Asamblea Mundial de la Salud, mediante la Resolución WHA62.14 de 2009, y en la Declaración Política de Río de Janeiro sobre determinantes sociales de la salud de 2011, mediante las cuales se insta a los Estados miembros a "que generen métodos y datos científicos nuevos, o utilicen los existentes, adaptándolos a los contextos nacionales con el fin de abordar los determinantes y los gradientes sociales de la salud, así como las inequidades sanitarias" [6], y al apoyo de la investigación para fundamentar las políticas y medidas, entre otros aspectos.

En Colombia, se han realizado esfuerzos en la caracterización de las condiciones sociodemográficas y de salud de las poblaciones y sus desigualdades. El Análisis de la Situación de Salud en Colombia 2002-2007, elaborado entre el Ministerio de la Protección Social y la Universidad de Antioquia en 2010 [7], dedica el tomo VI al análisis de desigualdades e inequidades. El Observatorio Nacional de Salud Pública dedica el $6^{\circ}$ Informe Técnico al estudio de las Desigualdades Sociales en Salud en el país, y describe en forma amplia los determinantes de las desigualdades sociales en salud y las desigualdades en salud en el país [8]. Posteriormente, y desde el año 2012, el país unificó la forma de presentación de los Análisis de Situación en Salud (ASIS) territoriales, con el propósito de generar comparabilidad en la información. En estos documentos, el abordaje al tema de determinantes sociales de la salud y la medición de desigualdades fue muy limitado, solo el componente de morbimortalidad tuvo un mayor abordaje, quedando pendiente la tarea de profundizar en desigualdades e inequidades regionales.

Para el presente trabajo, en la caracterización de las condiciones socioeconómicas se tomaron variables representativas de modelos de determinantes sociales y de producción de inequidades, según Whitehead, Brunner, Marmot y Wilkinson, Diderichsen y OMS [5], relacionadas con educación, economía, condiciones de ambiente y territorio. En lo referente a las condiciones sanitarias, se escogieron diversas mortalidades según los grupos de la Lista 6/67 OPS/OMS para la tabulación de mortalidad y la mortalidad en la niñez.

El objetivo del estudio es determinar las desigualdades en la mortalidad por causas específicas relacionadas con características socioeconómicas en municipios del Huila 2009-2013, con el propósito de aportar a un mejor y mayor abordaje de las desigualdades y de los determinantes sociales de la salud para el departamento.

\section{Metodología}

Es un estudio de tipo ecológico, con el objetivo de medir desigualdades sociales en las mortalidades por causas representativas de cada grupo, según la Lista de causas agrupadas de mortalidades 6/67 OMS/OPS, entre los 37 municipios del Huila, según el comportamiento de las variables socioeconómicas de 2009 a 2013.

Las causas representativas, por su importancia epidemiológica a nivel departamental, fueron:

- Infección Respiratoria Aguda (109),

- Tumor maligno de estómago (201),

- Enfermedades hipertensivas (302),

- Enfermedades cerebrovasculares (307),

- Accidentes de transporte terrestre (501),

- Agresiones -homicidios- (512) y

- Diabetes mellitus (601).

El código en paréntesis constituye el correspondiente para cada causa en la lista 6/67 de OMS. Adicionalmente, se describió la Mortalidad en la niñez -en menores de 5 años- (Mn), dada su importancia como indicador del nivel de salud de las poblaciones.

Las variables socioeconómicas seleccionadas fueron Cobertura en Educación Media (CEM 2013) para el 2013, Necesidades Básicas Insatisfechas del 2009 (NBI 2009), Valor Agregado Municipal del 2013 (VAM 2013), Índice de Riesgo de Calidad de Agua para Consumo Humano para el 2013 (IRCA 2013) y las variables Ruralidad y Suficiencia Económica del 2010.

La recolección de la información se realizó de fuentes secundarias (Tabla 1).

Los procedimientos para el cálculo de tasas y medidas de desigualdad se describen a continuación:

Tasa cruda: número de casos de cada mortalidad entre la población total del municipio, para cada año correspondiente, multiplicando por 100.000 .

Tasa ajustada: utilizando el método directo de ajuste de tasas, tomando como población de referencia a la población colombiana del censo 2005, tomando como grupos de edad 0 a 4 años, 5 a 14 años, 15 a 44 años, 45 a 64 años y 65 y más años.

Medidas basadas en rangos: se calculó el cociente y la diferencia de tasas (CT y DT) extremas por cuartiles y orden previo de cada variable, de la peor a la mejor situación socioeconómica.

- CT: Cociente de tasas extremas (Tasa Q4/Tasa Q1)

- DT: Diferencia de tasas extremas (Tasa Q4 - Tasa Q1)

Donde Q4 es el cuartil 4 (mejor situación socioeconómica) y Q1 el cuartil 1 (peor situación socioeconómica).

Para la variable ruralidad, las categorías son Eminentemente Rural + Rural $(\mathrm{ER}+\mathrm{R})$, Urbano Rural (UR), Urbano (U) y Eminentemente Urbano (EU). La categoría ER+R correspondería a Q1 y EU a Q4. 
Tabla 1. Relación de variables y fuentes de información

\begin{tabular}{|c|c|c|}
\hline Variable/año & Definición & Fuente de información y observaciones \\
\hline $\begin{array}{l}\text { Mortalidades } \\
2009 \text { a } 2013\end{array}$ & $\begin{array}{l}\text { Corresponde al número de muertes ocurrido anualmente } \\
\text { por cada causa definida }\end{array}$ & $\begin{array}{l}\text { Fuente: Departamento Administrativo Nacional de } \\
\text { Estadísticas (DANE). } \\
\text { La información se recopila con base en los Cuadros No. } \\
5 \text { de las defunciones no fetales de los años } 2009 \text { a 2013, } \\
\text { en donde se describe el número de muertes por sexo, } \\
\text { grupos de edad y causa según la lista } 6 / 67 \text { de la OPS } \\
\text { Adicionalmente, se identificó la mortalidad en menores } \\
\text { de } 5 \text { años para el cálculo de las tasas de mortalidad en la } \\
\text { niñez, para cada uno de los municipios del departamento }\end{array}$ \\
\hline $\begin{array}{l}\text { Cobertura en } \\
\text { Educación } \\
\text { Media } 2013\end{array}$ & $\begin{array}{l}\text { Porcentaje de estudiantes matriculados en educación } \\
\text { media (grados } 10 \text { y 11), sin contar los que están en } \\
\text { extra-edad }\end{array}$ & $\begin{array}{l}\text { Fuente: Departamento Nacional de Planeación (DNP) } \\
\text { Archivo Excel disponible en https://www. dnp.gov.co/ } \\
\text { programas/desarrollo-territorial/Estudios-Territoriales/ } \\
\text { Estudios-y-Ejercicios/Paginas/Brechas.aspx, link } \\
\text { Resultados brechas }\end{array}$ \\
\hline $\begin{array}{l}\text { NBI } \\
2011\end{array}$ & $\begin{array}{l}\text { Porcentaje de personas vs hogares sobre la población } \\
\text { total vs total de hogares que tienen al menos una } \\
\text { necesidad básica insatisfecha (viviendas con } \\
\text { hacinamiento crítico, con condiciones físicas impropias } \\
\text { para el alojamiento humano, servicios inadecuados, alta } \\
\text { dependencia económica o niños en edad escolar que no } \\
\text { asisten a la escuela) }\end{array}$ & $\begin{array}{l}\text { Fuente: DANE } \\
\text { Archivo Excel disponible en http://www.dane.gov.co/ } \\
\text { index.php/estadisticas-sociales/necesidades-basicas- } \\
\text { insatisfechas-nbi, link de Resultados Generales - } \\
\text { Necesidades básicas insatisfechas - desagregada }\end{array}$ \\
\hline $\begin{array}{l}\text { Ruralidad } \\
2010\end{array}$ & $\begin{array}{l}\text { Caracterización territorial basada en aspectos } \\
\text { demográficos, económicos y de uso del suelo, } \\
\text { tipificando las siguientes categorías: Eminentemente } \\
\text { Rural (ER), Rural (R), Urbano Rural (UR), Urbano (U) y } \\
\text { Eminentemente Urbano (EU). }\end{array}$ & $\begin{array}{l}\text { Fuente: Tesis de Maestría, Agrupación de municipios } \\
\text { colombianos según características de ruralidad [9] } \\
\text { Para la ruralidad se tomó como referencia la clasificación } \\
\text { descrita en el Anexo D del documento fuente }\end{array}$ \\
\hline $\begin{array}{l}\text { Suficiencia } \\
\text { Económica } \\
2010\end{array}$ & $\begin{array}{l}\text { Indicador socioeconómico que relaciona la Tasa Global } \\
\text { de Participación (TGP) y la Razón de Dependencia } \\
\text { Económica (RDE), cada una se categoriza en alta, } \\
\text { media o baja, y su cruce establece las categorías de } \\
\text { municipios económicamente equilibrados, suficientes y } \\
\text { muy insuficientes }\end{array}$ & $\begin{array}{l}\text { Fuente: Tesis de Maestría, Agrupación de municipios } \\
\text { colombianos según características de ruralidad [9] } \\
\text { En Suficiencia Económica se utilizó la clasificación } \\
\text { planteada en el Anexo C del documento fuente }\end{array}$ \\
\hline $\begin{array}{l}\text { Valor } \\
\text { Agregado } \\
\text { Municipal } \\
2013\end{array}$ & $\begin{array}{l}\text { Constituye el mayor valor creado en el proceso de } \\
\text { producción por efecto de la combinación de factores. Se } \\
\text { obtiene como diferencia entre el valor de la producción } \\
\text { bruta y los consumos intermedios empleados }\end{array}$ & $\begin{array}{l}\text { Fuente: Departamento Nacional de Planeación (DNP) } \\
\text { Archivo Excel disponible en https://ddtspr.dnp.gov.co/ } \\
\text { FIT/\#/fichas }\end{array}$ \\
\hline $\begin{array}{l}\text { IRCA } \\
2013\end{array}$ & $\begin{array}{l}\text { El grado de riesgo de ocurrencia de enfermedades } \\
\text { relacionadas con el no cumplimiento de las } \\
\text { características físicas, químicas y microbiológicas del } \\
\text { agua para consumo humano. La clasificación IRCA } \\
\text { (según Res. } 2115 \text { de 2007) es 0-5 sin riesgo; 5.1-14 } \\
\text { riesgo bajo; 14,1-35 riesgo medio; 35,1-80 riesgo alto; } \\
80.1-100 \text { inviable sanitariamente }\end{array}$ & $\begin{array}{l}\text { Fuente: Instituto Nacional de Salud (INS)/SIVICAP } \\
\text { Archivo Excel disponible en http://www.ins.gov.co/sivicap/ } \\
\text { Reportes/Reporte\%20IRCA\%20municipal_Huila.pdf }\end{array}$ \\
\hline
\end{tabular}

Y en la variable Suficiencia Económica las categorías fueron Económicamente Muy Insuficientes (EMI) correspondiente a Q1 y Económicamente Insuficientes (EI) representa a $\mathrm{Q} 4$.

Con base en las fórmulas, valores mayores que 1 en el CT y para la DT valores negativos indican mayor mortalidad en los municipios con peor situación socioeconómica.

Medidas basadas en regresión: se utilizaron los Índices de Desigualdad de la Pendiente (IDP) y el Índice Relativo de Desigualdad Acotado (IRDA), que permiten considerar el tamaño poblacional y la posición socioeconómica relativa de los grupos. El valor del IDP negativo indica mayor mortalidad en los municipios con la peor situación socioeconómica. Adicionalmente, se realizó el cálculo del coeficiente de Pearson y del coeficiente de determinación para establecer la condición de linealidad.

Medidas basadas en desproporcionalidad: se utilizó curva e índice de concentración. Cuando el índice toma valor negativo, se considera que existe una situación sanitaria adversa para los municipios menos favorecidos, y cuando los valores son muy cercanos a cero se considera que existe una situación muy cerca a la igualdad, sin desfavorecer o favorecer a ningún grupo social. 
Para el cálculo de las medidas de desigualdad se utilizaron las tasas ajustadas de las mortalidades por municipio como variables sanitarias, y se realizó el cruce con cada variable socioeconómica, ordenada de la peor a la mejor situación para el año correspondiente.

El análisis estadístico se realizó con el uso del software Excel 2010, y el análisis de las desigualdades fue realizado en Excel 2010 para el cálculo de las medidas de rango, y con Epidat 4.0 para el cálculo de las medidas de regresión y desproporcionalidad.

\section{Consideraciones éticas}

Este estudio responde a los aspectos éticos expresados en la Resolución número 008430 de 1993 del Ministerio de Salud de Colombia, por la cual se establecen las "Normas Científicas, Técnicas y Administrativas para la Investigación en Salud". Esta investigación es considerada "sin riesgo" según el artículo 11, literal A. No se realiza intervención o modificación intencionada de las variables fisiológicas, biológicas ni psicológicas o sociales, puesto que para la recolección de la información se utilizaron fuentes secundarias tales como registros y bases de datos. Para ello se utilizó un instrumento que permitió optimizar la recolección de la información, siendo avalado por el Comité de Ética de la Universidad Autónoma de Manizales.

\section{Resultados}

Los resultados se presentan en tres partes: comportamiento de las mortalidades, comportamiento de las variables socioeconómicas y medición de desigualdades.

\section{Comportamiento de la mortalidad}

En la Tabla 2 se presenta el comportamiento de casos y tasas por evento a nivel departamental, y en la Tabla 3 se resume el comportamiento municipal por cuartiles.

La Infección Respiratoria Aguda anualmente aporta cerca de 150 muertes en el Huila, con distribución muy similar entre hombres y mujeres. El año 2011 fue el de mejor comportamiento, con 13 municipios sin presencia de casos y una tasa departamental de 10,95 x 100.000 habitantes.

El tumor maligno de estómago es la principal causa de muerte por neoplasia maligna en el departamento, anualmente fallecen cerca de 150 personas, para una tasa cruda promedio durante el periodo de 13,86 por 100.000 habitantes. Durante el año 2010 se presentó la menor tasa del periodo, con 12,79 muertes por cada 100.000 habitantes y un total de 140 casos.

Las enfermedades hipertensivas son causa de aproximadamente 140 muertes al año y presentan una ligera mayor incidencia en mujeres. La tasa cruda promedio departamental de 2009 a 2013 fue 13,16 por
100.000 habitantes. La mortalidad por enfermedades cerebrovasculares alcanza cifras cercanas a las 30 muertes por 100.000 habitantes, con un promedio cercano a las 310 muertes anuales.

Los accidentes de transporte terrestre constituyen la segunda causa de muerte por lesión de causa externa, superada por los homicidios. En el departamento, se presentan alrededor de 210 muertes anuales, y los hombres aportan 5 a 4 muertes por cada mortalidad en mujeres. El municipio de Neiva evidenció una mejoría gradual y progresiva en sus tasas de mortalidad por accidentes de tránsito, pasando de 24 a 15 por 100.000 habitantes.

De los cinco años observados, el año 2009 fue el que evidenció mayores tasas de homicidio, con 35,14 por 100.000 habitantes. De los cuatro municipios principales del departamento, Pitalito aporta la mayor tasa promedio del periodo, con 41 por 100.000 habitantes. Por el contrario, Garzón presenta tasa promedio de 19 por 100.000 habitantes, muy inferior al promedio departamental de 29 homicidios por 100.000 habitantes, y Neiva una tasa promedio de 30 por 100.000 habitantes.

La diabetes mellitus ocasiona cerca de 170 muertes en promedio anual en el Huila, alcanzando una tasa de mortalidad de 15 por 100.000 habitantes. En los municipios de Neiva y Pitalito se presentaron tasas promedio de 18 muertes por 100.000 habitantes, y en la Plata, principal municipio de la zona occidente y el más rural de los principales municipios, una tasa de 10 por 100.000 habitantes

A nivel departamental, se presentaron en promedio 270 muertes anuales en menores de 5 años, siendo 2009 el de mayor aporte, con un total de 312 muertes, y el año 2011 el de mejor comportamiento, con 245 fallecimientos en esta población. La tasa cruda promedio departamental fue 13,44 muertes por 1.000 nacidos vivos, con rango entre 15,78 en 2011 y 13,25 para 2013.

\section{Descripción de las variables socioeconómicas}

El comportamiento de las variables se presenta en forma consolidada en la Tabla 4, con agrupación por cuartiles desde la peor a la mejor situación socioeconómica.

En el departamento del Huila, 11 municipios tienen una cobertura neta en educación media menor al 30\% y el municipio con menor cobertura es Colombia, con el $14,45 \%$. Solo $24 \%$ de los municipios tiene una cobertura neta en educación media mayor al $41.3 \%$ para el año 2013. Un $27 \%$ de los municipios tiene un NBI mayor a $44.9 \%$, donde el municipio con mayor NBI es Colombia, con $64.77 \%$. En el cuartil de mejor comportamiento, con un NBI menor a $31.9 \%$ se ubican nueve municipios. Neiva (17,7\%), Yaguará (21.84\%) y Altamira (23,6\%) son los municipios con mejores condiciones. Frente al VAM, 10 municipios (27\%) tienen un valor menor a 
Tabla 2. Casos y tasas ajustadas de mortalidades. Huila 2009 a 2013

\begin{tabular}{|c|c|c|c|c|c|c|c|c|c|c|}
\hline \multirow{2}{*}{ Evento } & \multicolumn{2}{|c|}{2009} & \multicolumn{2}{|c|}{2010} & \multicolumn{2}{|c|}{2011} & \multicolumn{2}{|c|}{2012} & \multicolumn{2}{|c|}{2013} \\
\hline & Muertes & Tasa & Muertes & Tasa & Muertes & Tasa & Muertes & Tasa & Muertes & Tasa \\
\hline $\begin{array}{l}\text { Infección } \\
\text { Respiratoria Aguda }\end{array}$ & 139 & 12,98 & 140 & 12,76 & 123 & 10,95 & 178 & 15,49 & 182 & 15,46 \\
\hline $\begin{array}{l}\text { Tumor maligno de } \\
\text { estómago }\end{array}$ & 161 & 15,07 & 140 & 12,79 & 150 & 13,3 & 154 & 13,29 & 153 & 12,89 \\
\hline $\begin{array}{l}\text { Enfermedades } \\
\text { hipertensivas }\end{array}$ & 121 & 11,33 & 156 & 14,23 & 133 & 11,8 & 162 & 14,04 & 151 & 12,7 \\
\hline $\begin{array}{l}\text { Enfermedades } \\
\text { cerebrovasculares }\end{array}$ & 319 & 29,87 & 305 & 27,8 & 278 & 24,69 & 329 & 28,51 & 336 & 28,27 \\
\hline $\begin{array}{l}\text { Accidentes de } \\
\text { tránsito }\end{array}$ & 223 & 21,19 & 234 & 21,86 & 194 & 17,78 & 208 & 18,69 & 222 & 19,53 \\
\hline $\begin{array}{l}\text { Agresiones } \\
\text { (homicidios) }\end{array}$ & 367 & 35,14 & 311 & 29,17 & 279 & 25,7 & 301 & 27,14 & 313 & 27,66 \\
\hline Diabetes mellitus & 164 & 15,34 & 169 & 15,6 & 178 & 16,22 & 161 & 14,48 & 158 & 14,03 \\
\hline $\begin{array}{l}\text { Mortalidad en la } \\
\text { niñez }\end{array}$ & 312 & 15,35 & 251 & 15,38 & 245 & 15,78 & 280 & 13,92 & 257 & 13,25 \\
\hline
\end{tabular}

Nota: todas las tasas se presentan en valores de número de muertes por cada 100.000 habitantes, excepto en la mortalidad en la niñez, en donde se presenta como número de muertes en menores de 5 años por cada 1.000 nacidos vivos.

Tabla 3. Promedio y desviación por cuartiles de las tasas ajustadas de mortalidad por edad y por año. Huila y municipios, 2009 a 2013

\begin{tabular}{|c|c|c|c|c|c|c|c|c|c|c|}
\hline \multirow[t]{2}{*}{ Cuartiles/Evento } & \multicolumn{2}{|c|}{2009} & \multicolumn{2}{|c|}{2010} & \multicolumn{2}{|c|}{2011} & \multicolumn{2}{|c|}{2012} & \multicolumn{2}{|c|}{2013} \\
\hline & Media & $\mathrm{DE}$ & Media & $\mathrm{DE}$ & Media & $\mathrm{DE}$ & Media & DE & Media & $\mathrm{DE}$ \\
\hline \multicolumn{11}{|c|}{ Infección Respiratoria Aguda } \\
\hline Q1 & 25,37 & 4,50 & 23,85 & 5,97 & 17,43 & 3,60 & 21,40 & 5,67 & 27,05 & 24,29 \\
\hline Q2 & 14,92 & 3,06 & 14,29 & 1,82 & 10,58 & 1,88 & 13,65 & 0,88 & 11,63 & 2,05 \\
\hline Q3 & 7,92 & 1,55 & 8,48 & 1,26 & 6,01 & 0,51 & 9,14 & 2,72 & 6,70 & 1,59 \\
\hline Q4 & 0,32 & 0,96 & 1,47 & 2,24 & 0,00 & 0,00 & 0,00 & 0,00 & 0,00 & 0,00 \\
\hline \multicolumn{11}{|c|}{ Tumor maligno de estómago } \\
\hline Q1 & 28,94 & 13,80 & 23,01 & 5,73 & 24,54 & 7,58 & 29,98 & 12,23 & 31,20 & 8,47 \\
\hline Q2 & 15,91 & 1,33 & 14,15 & 2,08 & 14,72 & 2,13 & 12,41 & 1,61 & 17,78 & 1,99 \\
\hline Q3 & 9,44 & 2,98 & 10,14 & 0,82 & 8,52 & 1,74 & 7,38 & 1,42 & 11,90 & 1,64 \\
\hline Q4 & 0,00 & 0,00 & 2,49 & 3,77 & 0,37 & 1,11 & 0,49 & 1,46 & 4,29 & 3,53 \\
\hline \multicolumn{11}{|c|}{ Enfermedades hipertensivas } \\
\hline Q1 & 30,58 & 8,10 & 32,88 & 4,84 & 42,81 & 15,76 & 40,48 & 18,36 & 37,06 & 13,01 \\
\hline Q2 & 17,17 & 2,56 & 20,19 & 4,13 & 17,78 & 2,98 & 13,74 & 2,61 & 17,87 & 1,87 \\
\hline Q3 & 9,43 & 1,64 & 10,73 & 1,92 & 7,89 & 3,39 & 8,76 & 1,52 & 9,82 & 2,57 \\
\hline Q4 & 0,79 & 2,37 & 2,17 & 3,28 & 0,00 & 0,00 & 0,97 & 2,02 & 1,00 & 2,12 \\
\hline \multicolumn{11}{|c|}{ Enfermedades cerebrovasculares } \\
\hline Q1 & 56,25 & 13,23 & 50,49 & 24,48 & 39,66 & 9,35 & 44,61 & 11,05 & 48,81 & 8,73 \\
\hline Q2 & 30,79 & 3,63 & 29,84 & 2,94 & 29,18 & 3,46 & 29,19 & 2,52 & 31,38 & 2,89 \\
\hline Q3 & 21,76 & 2,52 & 19,63 & 3,47 & 16,62 & 3,61 & 18,28 & 3,21 & 20,18 & 3,82 \\
\hline Q4 & 12,49 & 2,42 & 8,49 & 5,34 & 7,29 & 4,61 & 5,31 & 4,17 & 5,61 & 5,33 \\
\hline \multicolumn{11}{|c|}{ Accidentes de tránsito } \\
\hline Q1 & 43,11 & 18,43 & 38,72 & 7,94 & 35,81 & 9,95 & 32,01 & 10,38 & 47,45 & 19,80 \\
\hline Q2 & 24,53 & 3,08 & 25,26 & 3,97 & 22,00 & 2,55 & 20,76 & 2,67 & 20,97 & 3,83 \\
\hline
\end{tabular}


Continuación tabla 3

\begin{tabular}{|c|c|c|c|c|c|c|c|c|c|c|}
\hline \multirow[t]{2}{*}{ Cuartiles/Evento } & \multicolumn{2}{|c|}{2009} & \multicolumn{2}{|c|}{2010} & \multicolumn{2}{|c|}{2011} & \multicolumn{2}{|c|}{2012} & \multicolumn{2}{|c|}{2013} \\
\hline & Media & DE & Media & DE & Media & DE & Media & DE & Media & DE \\
\hline Q3 & 13,35 & 3,09 & 15,08 & 3,41 & 14,25 & 3,38 & 11,71 & 3,46 & 11,33 & 2,45 \\
\hline Q4 & 3,54 & 4,34 & 2,15 & 4,30 & 2,90 & 3,68 & 0,50 & 1,49 & 3,38 & 3,38 \\
\hline \multicolumn{11}{|c|}{ Agresiones (homicidios) } \\
\hline Q1 & 64,62 & 9,71 & 55,66 & 21,55 & 44,26 & 11,17 & 44,22 & 11,41 & 46,84 & 9,19 \\
\hline Q2 & 39,60 & 8,14 & 32,84 & 6,17 & 27,64 & 4,09 & 26,50 & 3,11 & 30,23 & 2,08 \\
\hline Q3 & 24,48 & 6,09 & 15,17 & 4,01 & 15,06 & 2,66 & 16,66 & 3,41 & 16,97 & 5,42 \\
\hline Q4 & 8,70 & 5,19 & 4,38 & 4,36 & 5,47 & 4,48 & 2,83 & 4,27 & 6,05 & 3,63 \\
\hline \multicolumn{11}{|c|}{ Diabetes mellitus } \\
\hline Q1 & 29,64 & 14,21 & 27,47 & 10,20 & 34,87 & 16,04 & 29,30 & 9,04 & 31,91 & 25,99 \\
\hline Q2 & 12,72 & 1,76 & 15,01 & 2,32 & 16,81 & 1,78 & 14,72 & 1,62 & 15,66 & 2,25 \\
\hline Q3 & 7,21 & 3,31 & 9,77 & 1,61 & 9,82 & 1,95 & 8,76 & 2,37 & 10,98 & 1,91 \\
\hline Q4 & 0,00 & 0,00 & 1,87 & 2,87 & 2,49 & 3,13 & 0,54 & 1,62 & 2,77 & 3,44 \\
\hline \multicolumn{11}{|c|}{ Mortalidad en la niñez } \\
\hline Q1 & 26,42 & 3,32 & 21,87 & 2,21 & 30,94 & 9,88 & 20,35 & 2,98 & 23,70 & 10,09 \\
\hline Q2 & 18,51 & 1,24 & 17,30 & 1,22 & 14,66 & 1,58 & 15,56 & 0,69 & 13,21 & 1,11 \\
\hline Q3 & 14,19 & 1,88 & 12,77 & 1,12 & 10,26 & 1,08 & 11,34 & 1,58 & 10,07 & 1,14 \\
\hline Q4 & 7,71 & 3,67 & 7,72 & 1,76 & 5,09 & 2,77 & 6,62 & 3,09 & 5,57 & 2,61 \\
\hline
\end{tabular}

Tabla 4. Distribución por cuartiles de las variables socioeconómicas Cobertura en Educación Media, NBI, Valor agregado municipal e IRCA. Municipios del Huila 2009 a 2013.

\begin{tabular}{|c|c|c|c|c|c|c|c|c|}
\hline Cuartil & Municipio & $\begin{array}{l}\text { CME } \\
2013\end{array}$ & Municipio & NBI 2009 & Municipio & VAM 2013 & Municipio & IRCA 2013 \\
\hline \multirow{10}{*}{ Q1 } & Colombia & $14,5 \%$ & Colombia & 64,8 & Palestina & $\$ 1.193 .690$ & Oporapa & 80,0 \\
\hline & Baraya & $22,0 \%$ & Acevedo & 64,1 & Colombia & $\$ 1.335 .266$ & Elías & 72,0 \\
\hline & Acevedo & $22,4 \%$ & Tello & 53,5 & Nátaga & $\$ 1.432 .961$ & Pital & 72,0 \\
\hline & Aipe & $24,9 \%$ & Oporapa & 53,3 & Oporapa & $\$ 1.443 .914$ & Guadalupe & 71,0 \\
\hline & Teruel & $25,6 \%$ & Saladoblanco & 49,4 & Isnos & $\$ 1.448 .749$ & Santa María & 70,0 \\
\hline & Oporapa & $26,0 \%$ & Algeciras & 49,2 & Acevedo & $\$ 1.513 .127$ & Colombia & 70,0 \\
\hline & Suaza & $27,7 \%$ & Isnos & 46,6 & La Argentina & $\$ 1.516 .543$ & Suaza & 70,0 \\
\hline & Tello & $28,4 \%$ & Suaza & 45,7 & Guadalupe & $\$ 1.535 .763$ & Aipe & 69,0 \\
\hline & Elías & $29,3 \%$ & Palestina & 45,0 & Tarqui & $\$ 1.610 .997$ & Saladoblanco & 67,0 \\
\hline & Saladoblanco & $29,5 \%$ & Agrado & 44,9 & Suaza & $\$ 1.682 .293$ & La Argentina & 66,0 \\
\hline \multirow{9}{*}{ Q2 } & Iquira & $30,0 \%$ & Pital & 44,9 & Gigante & $\$ 1.699 .635$ & Agrado & 65,0 \\
\hline & Villavieja & $32,1 \%$ & La Argentina & 44,7 & Saladoblanco & $\$ 1.711 .098$ & Timaná & 65,0 \\
\hline & Garzón & $32,3 \%$ & La Plata & 44,5 & San Agustín & $\$ 1.735 .547$ & Nátaga & 64,0 \\
\hline & Palestina & $33,0 \%$ & Iquira & 44,3 & Algeciras & $\$ 1.735 .803$ & Acevedo & 63,0 \\
\hline & Campoalegre & $33,3 \%$ & San Agustín & 42,5 & Elías & $\$ 1.758 .696$ & Tesalia & 62,0 \\
\hline & Palermo & $34,2 \%$ & Nátaga & 42,3 & La Plata & $\$ 1.822 .870$ & San Agustín & 61,0 \\
\hline & Algeciras & $34,9 \%$ & Santa María & 42,0 & Iquira & $\$ 1.851 .115$ & Pitalito & 61,0 \\
\hline & Altamira & $35,3 \%$ & Tarqui & 40,9 & Teruel & $\$ 1.852 .997$ & La Plata & 60,0 \\
\hline & Hobo & $35,3 \%$ & Villavieja & 40,0 & Garzón & $\$ 1.899 .459$ & lquira & 59,0 \\
\hline
\end{tabular}




\begin{tabular}{|c|c|c|c|c|c|c|c|c|}
\hline Cuartil & Municipio & $\begin{array}{l}\text { CME } \\
2013 \\
\end{array}$ & Municipio & NBI 2009 & Municipio & VAM 2013 & Municipio & IRCA 2013 \\
\hline \multirow{9}{*}{ Q3 } & La Plata & $35,4 \%$ & Baraya & 39,7 & Tello & $\$ 1.929 .539$ & Palestina & 59,0 \\
\hline & Pital & $37,1 \%$ & Paicol & 38,4 & Pital & $\$ 1.939 .755$ & Paicol & 59,0 \\
\hline & Agrado & $37,2 \%$ & Aipe & 38,0 & Timaná & $\$ 1.969 .274$ & Algeciras & 56,0 \\
\hline & Timaná & $37,4 \%$ & Pitalito & 37,1 & Santa María & $\$ 2.068 .538$ & Teruel & 55,0 \\
\hline & Guadalupe & $37,6 \%$ & Guadalupe & 36,9 & Aipe & $\$ 2.129 .358$ & Yaguará & 55,0 \\
\hline & Gigante & $38,6 \%$ & Teruel & 34,5 & Villavieja & $\$ 2.174 .044$ & Baraya & 55,0 \\
\hline & La Argentina & $40,4 \%$ & Elías & 33,3 & Altamira & $\$ 2.211 .107$ & Altamira & 55,0 \\
\hline & Paicol & $41,1 \%$ & Hobo & 32,7 & Agrado & $\$ 2.265 .252$ & Campoalegre & 53,0 \\
\hline & Isnos & $41,2 \%$ & Rivera & 32,3 & Baraya & $\$ 2.277 .851$ & Tello & 51,0 \\
\hline \multirow{9}{*}{ Q4 } & Tarqui & $41,5 \%$ & Timaná & 31,5 & Rivera & $\$ 2.328 .989$ & Hobo & 50,0 \\
\hline & Pitalito & $42,8 \%$ & Garzón & 30,9 & Campoalegre & $\$ 2.347 .268$ & Garzón & 49,0 \\
\hline & San Agustín & $43,1 \%$ & Gigante & 30,7 & Hobo & $\$ 2.371 .017$ & Tarqui & 48,0 \\
\hline & Santa María & $43,8 \%$ & Palermo & 30,2 & Pitalito & $\$ 2.508 .471$ & Gigante & 48,0 \\
\hline & Neiva & $47,8 \%$ & Tesalia & 29,5 & Paicol & $\$ 2.581 .638$ & Isnos & 43,0 \\
\hline & Yaguará & $47,9 \%$ & Campoalegre & 26,7 & Tesalia & $\$ 2.702 .536$ & Palermo & 35,0 \\
\hline & Nátaga & $49,1 \%$ & Altamira & 23,6 & Palermo & $\$ 3.035 .225$ & Rivera & 31,0 \\
\hline & Rivera & $52,8 \%$ & Yaguará & 21,8 & Neiva & $\$ 3.712 .605$ & Neiva & 29,0 \\
\hline & Tesalia & $53,5 \%$ & Neiva & 17,7 & Yaguará & $\$ 3.733 .722$ & Villavieja & 3,0 \\
\hline
\end{tabular}

$\$ 1.682 .293$ y $24 \%$ de los municipios (9) tiene un valor mayor a \$2.328.989 en orden ascendente, como son: Rivera, Campoalegre, Hobo, Pitalito, Paicol, Tesalia, Palermo, Neiva y el último con mayor valor Yaguará (\$3.733.722 de VAM).

En el año 2013, el municipio de Villavieja presenta una clasificación del IRCA sin riesgo (menor a 5), tres municipios presentan riesgo medio (Neiva, Palermo y Rivera) y los 33 municipios restantes obtuvieron un IRCA dentro del rango de riesgo alto (IRCA entre 35,1 y 80). Un total de 11 municipios son Urbano Rurales y 30\% son Rurales y Eminentemente Rurales, con una configuración territorial con espacios de alto contenido natural. Un $84 \%$ de los municipios (31 de 37) del departamento del Huila se clasifican como económicamente muy insuficientes, cuentan con una Razón de Dependencia Económica (RDE) alta y una baja Tasa Global de Participación (TGP), y el restante son económicamente insuficientes, caracterizados por RDE alta con media TGP, o con RDE media y baja TGP.

\section{Medición de desigualdades}

Se presentan en el siguiente orden: medidas basadas en rango (CT y DT), medidas basadas en regresión (Índice y curva de concentración) y medidas basadas en desproporcionalidad (IDP e IRDA).

\section{Medidas basadas en rango}

En la tabla 5 se presenta el resumen de las DT y CT de cada variable socioeconómica, frente a las mortalidades en estudio.

Las tasas de mortalidad por tumor maligno de estómago, enfermedades hipertensivas y en la niñez son más altas en los municipios con más baja Cobertura en Educación Media, en donde fallecen casi dos veces más (CT 0,55) personas por enfermedades hipertensivas que en los municipios con mejor cobertura. Por el contrario, la Infección Respiratoria Aguda causa 11 muertes más por cada 100.000 habitantes en los municipios con mejor cobertura, frente a los municipios con más baja cobertura.

En los municipios con mayores porcentajes de NBI, las tasas de mortalidad por enfermedades hipertensivas, homicidios y en Mortalidad en la niñez son más altas; por ejemplo, fallecen cerca del doble de personas (CT 0,51) por enfermedades hipertensivas frente a los municipios con menores porcentajes de NBI, y se observa en homicidios un exceso de 17 muertes por 100.000 habitantes frente a los municipios con mejor NBI. Por el contrario, en los municipios con menores porcentajes NBI se observan mayores tasas en las mortalidades por Infección Respiratoria Aguda, enfermedades cerebrovasculares, accidentes de tránsito y diabetes mellitus. La accidentalidad 
Tabla 5. Desigualdades en la mortalidad según medidas basadas en rango, en los municipios del Huila 2009 a 2013

\begin{tabular}{|c|c|c|c|c|c|c|c|c|c|}
\hline \multirow{2}{*}{$\begin{array}{c}\text { Variable } \\
\text { socioeconómica }\end{array}$} & \multirow{2}{*}{$\begin{array}{c}\text { Medida } \\
\text { de } \\
\text { rango }\end{array}$} & \multicolumn{8}{|c|}{ Códigos de mortalidades - Lista 6/67 (OMS/OPS) } \\
\hline & & 109 & 201 & 302 & 307 & 501 & 512 & 601 & $\mathrm{Mn}$ \\
\hline \multirow{2}{*}{ CEM 2013} & CT & 2,50 & 0,50 & 0,74 & 1,09 & 1,01 & 1,15 & 1,35 & 0,86 \\
\hline & DT & 10,97 & $-10,32$ & $-4,78$ & 2,58 & 0,18 & 4,35 & 3,5 & $-1,93$ \\
\hline \multirow{2}{*}{ NBI 2009} & CT & 1,39 & 1,00 & 0,51 & 1,35 & 1,75 & 0,64 & 1,32 & 0,70 \\
\hline & DT & 4,16 & $-0,08$ & -10 & 8,66 & 9,92 & $-17,06$ & 4,11 & $-6,22$ \\
\hline \multirow{2}{*}{ Ruralidad 2010} & CT & 0,79 & 0,98 & 0,53 & 1,05 & 1,02 & 0,99 & 0,91 & 1,00 \\
\hline & DT & $-3,48$ & $-0,29$ & $-11,21$ & 1,3 & 0,43 & $-0,43$ & $-1,68$ & $-0,05$ \\
\hline \multirow{2}{*}{ SE 2010} & CT & 1,20 & 0,85 & 0,70 & 1,08 & 0,93 & 0,85 & 1,47 & 0,93 \\
\hline & DT & 2,49 & $-2,14$ & $-5,17$ & 1,99 & $-1,73$ & $-4,72$ & 13,23 & $-1,02$ \\
\hline \multirow{2}{*}{ VAM 2013} & CT & 2,08 & 0,43 & 0,66 & 1,03 & 1,32 & 1,08 & 1,14 & 1,02 \\
\hline & DT & 9,91 & $-15,1$ & $-6,35$ & 0,99 & 5,09 & 2,23 & 1,66 & 0,27 \\
\hline \multirow{2}{*}{ IRCA 2013} & CT & 0,45 & 1,74 & 1,77 & 1,09 & 0,71 & 0,97 & 1,01 & 1,16 \\
\hline & DT & 2,22 & 0,57 & 0,56 & 0,92 & 1,41 & 1,03 & 0,99 & 0,86 \\
\hline
\end{tabular}

${ }^{a}$ CT: Cociente de tasas; DT: Diferencia de tasas

en tránsito causa cerca de 10 muertes más por cada 100.000 habitantes en los municipios con mejor NBI, frente a los municipios con peor NBI.

Hubo mayor mortalidad en el cuartil más rural en las causas por enfermedades hipertensivas, seguida por la Infección Respiratoria Aguda. Se evidencian cocientes de tasas extremas muy cercanos a la unidad en la mayoría de las mortalidades, incluida la Mortalidad en la niñez.

Se observaron desigualdades adversas para el grupo de municipios Económicamente Muy Insuficientes en las mortalidades por tumor maligno de estómago, enfermedades hipertensivas, accidentes de tránsito y homicidios, y en la Mortalidad en la niñez. La mayor diferencia de tasas de mortalidad se evidencia en diabetes mellitus, en donde los municipios Económicamente Insuficientes presentan 13 muertes por 100.000 habitantes más que los municipios Económicamente Muy Insuficientes.

Las mortalidades por tumor maligno del estómago y enfermedades hipertensivas presentan cerca de 15 y 6 muertes adicionales, respectivamente, por cada 100.000 habitantes en los municipios del cuartil con más bajo VAM. Situación contraria se presenta en la mortalidad por Infección Respiratoria Aguda, en donde se presenta el doble de muertes $($ CT 0,48$)$ en el cuartil de municipios con mayor VAM y en accidentes de tránsito, con un 30\% más de muertes en los mismos municipios respecto al cuartil de menor VAM.

\section{Medidas basadas en regresión: IDP e IRDA}

Para las medidas basadas en regresión, el análisis se realiza solo con las variables cuantitativas, excluyendo
Ruralidad y Suficiencia Económica. El resumen de los índices de regresión se presenta en la Tabla 6.

Para el NBI en el año 2009, el valor más alto del $\mathrm{R}^{2}$ alcanza un $12,1 \%$ en la accidentalidad en tránsito, con un valor de $\mathrm{r}$ por encima del valor crítico de 0,275 , sugiriendo linealidad del modelo con situación adversa para los municipios más favorecidos con un IDP de 28,23. Por el contrario, en los homicidios, con un IDP de -34,13 y un $r$ de -0,3491, sugiere asociación lineal en contra de los menos favorecidos, como se observa en la Figura 1.

En el análisis desde el valor agregado municipal, solo se observó asociación con la mortalidad por tumor maligno de estómago, con una IDP de -11,66 y un $\mathrm{r}$ de $-0,2937$. Se sugiere explicación lineal en el comportamiento de las variables con mayores tasas de mortalidad en los municipios con menor VAM.

Medidas basadas en desproporcionalidad: indice y curva de concentración.

En la Tabla 7 se presenta el resumen de los índices y en la Figura 2 las curvas con mayor valor de los índices de concentración.

Al observar el comportamiento de la mortalidad según la Cobertura en Educación Media, los índices negativos se observan en mortalidad por tumor maligno de estómago, en donde el $50 \%$ de los municipios con más baja cobertura aportan el $60 \%$ de las muertes. Situación contraria se evidencia en los homicidios, con un índice positivo, y donde el $50 \%$ de la población con menores coberturas comprende $40 \%$ de las muertes por estas causas (Tabla 7). Las demás mortalidades reflejan 
Tabla 6. Desigualdades en la mortalidad según medidas basadas en regresión, en los municipios del Huila 2009 a 2013.

\begin{tabular}{|c|c|c|c|c|c|c|c|c|c|}
\hline \multirow{2}{*}{$\begin{array}{c}\text { Variables } \\
\text { socioeconómicas }\end{array}$} & \multirow{2}{*}{$\begin{array}{l}\text { Índices de } \\
\text { regresión* }\end{array}$} & \multicolumn{8}{|c|}{ Códigos Lista 6/67 de las mortalidades } \\
\hline & & 109 & 201 & 302 & 307 & 501 & 512 & 601 & $\mathrm{Mn}$ \\
\hline \multirow{4}{*}{$\begin{array}{l}\text { CEM } \\
2013\end{array}$} & IDP & 2,45 & $-5,62$ & 1,68 & 7,47 & 30,82 & 7,41 & 1,81 & $-4,27$ \\
\hline & IRDA & 0,84 & 1,41 & 0,92 & 0,78 & 0,26 & 0,75 & 0,9 & 1,4 \\
\hline & r & 0,0567 & $-0,176$ & 0,0186 & 0,1485 & 0,4756 & 0,1434 & 0,0236 & $-0,154$ \\
\hline & $R^{2}$ & $0,30 \%$ & $3,10 \%$ & $0,00 \%$ & $2,20 \%$ & $21,30 \%$ & $1,20 \%$ & $0,10 \%$ & $2,40 \%$ \\
\hline \multirow{4}{*}{$\begin{array}{l}N B \mid \\
2009\end{array}$} & IDP & 5,01 & $-7,43$ & $-5,75$ & 20,57 & 28,23 & $-34,13$ & 3,19 & $-8,12$ \\
\hline & IRDA & 0,74 & 1,49 & 1,31 & 0,56 & 0,35 & 2,64 & 0,84 & 1,52 \\
\hline & $r$ & 0,1433 & $-0,1565$ & $-0,153$ & 0,2635 & 0,3738 & $-0,3491$ & 0,0619 & $-0,2567$ \\
\hline & $R^{2}$ & $2,10 \%$ & $2,40 \%$ & $3,00 \%$ & $6,90 \%$ & $12,10 \%$ & $8,30 \%$ & $0,40 \%$ & $6,60 \%$ \\
\hline \multirow{4}{*}{$\begin{array}{l}\text { VAM } \\
2013\end{array}$} & IDP & 9,91 & $-11,66$ & 0,84 & $-5,92$ & 17,17 & $-11,01$ & 9,83 & 3,08 \\
\hline & IRDA & 0,52 & 2,21 & 0,96 & 1,24 & 0,49 & 1,6 & 0,58 & 0,8 \\
\hline & r & 0,1667 & $-0,2937$ & $-0,006$ & $-0,0908$ & 0,2149 & $-0,1582$ & 0,1377 & 0,0792 \\
\hline & $\mathrm{R}^{2}$ & $2,80 \%$ & $8,60 \%$ & $0,00 \%$ & $0,80 \%$ & $3,70 \%$ & $3,30 \%$ & $1,90 \%$ & $0,60 \%$ \\
\hline \multirow{4}{*}{$\begin{array}{l}\text { IRCA } \\
2013\end{array}$} & IDP & 8,14 & $-3,13$ & $-7,86$ & $-12,26$ & 7,75 & $-3,91$ & 6,63 & $-3,93$ \\
\hline & IRDA & 0,58 & 1,21 & 1,56 & 1,59 & 0,72 & 1,17 & 0,69 & 1,36 \\
\hline & $r$ & 0,1591 & $-0,0765$ & $-0,1603$ & $-0,1826$ & 0,0997 & $-0,0415$ & 0,1015 & $-0,1185$ \\
\hline & $R^{2}$ & $2,50 \%$ & $0,60 \%$ & $0,30 \%$ & $3,30 \%$ & $0,80 \%$ & $0,10 \%$ & $1,00 \%$ & $1,40 \%$ \\
\hline
\end{tabular}

* IDP: Índice de Desigualdad de la Pendiente; IRDA: Índice Relativo de Desigualdad Acotado; r: Coeficiente de Pearson; R²: Coeficiente de determinación

Nota: Infección Respiratoria Aguda (109), tumor maligno de estómago (201), enfermedades hipertensivas (302), enfermedades cerebrovasculares (307), accidentes de transporte terrestre (501), agresiones -homicidios- (512), diabetes mellitus (601), Mortalidad en la niñez (Mn).
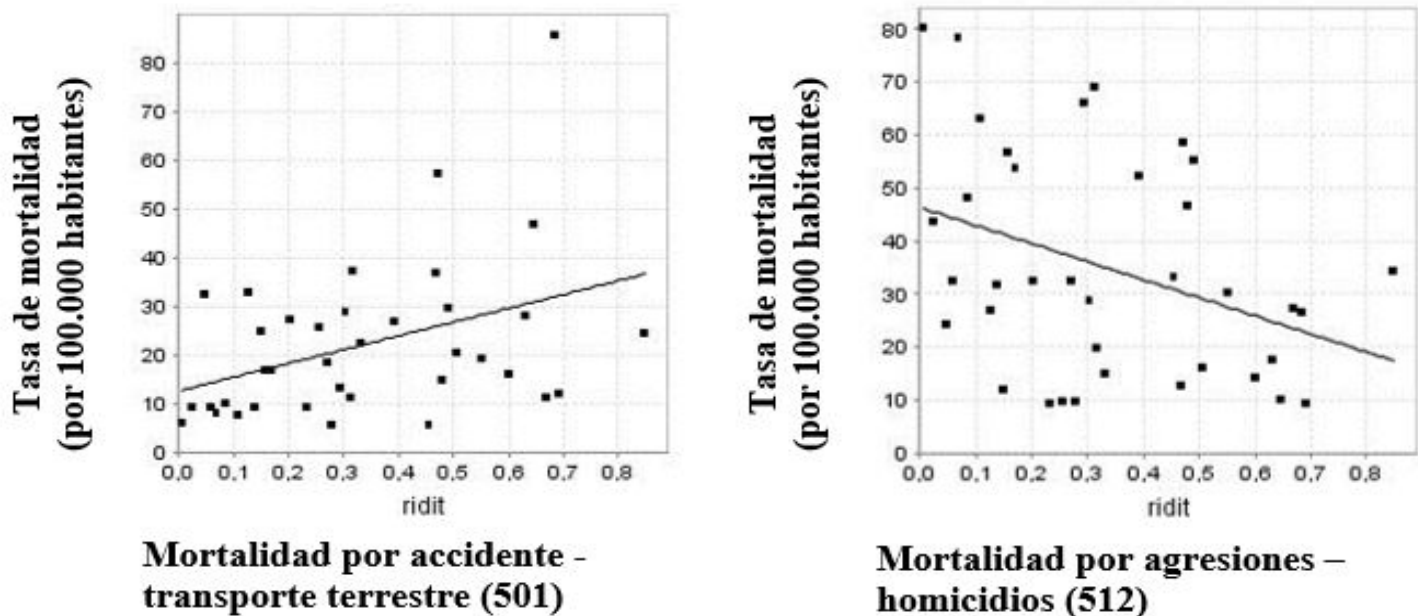

Mortalidad por agresiones -
homicidios (512)

Figura 1. Tasa de mortalidad por accidentes de tránsito y homicidios, según la posición relativa acumulada de la población, ordenada en función de NBI en los municipios del Huila en 2009 
Tabla 7. Índices de concentración en las desigualdades en la mortalidad según medidas basadas en desproporcionalidad, en los municipios del Huila 2009 a 2013

\begin{tabular}{lcccccccc}
\hline \multirow{2}{*}{$\begin{array}{c}\text { Variables } \\
\text { socioeconómicas }\end{array}$} & $\mathbf{7}$ Códigos de mortalidades - Lista 6/67 (OMS/OPS) \\
\cline { 2 - 9 } & $\mathbf{1 0 9}$ & $\mathbf{2 0 1}$ & $\mathbf{3 0 2}$ & $\mathbf{3 0 7}$ & $\mathbf{5 0 1}$ & $\mathbf{5 1 2}$ & $\mathbf{6 0 1}$ & $\mathbf{M n}$ \\
\hline CEM 2013 & 0,1126 & $-0,0978$ & $-0,0567$ & 0,243 & 0,0186 & 0,0847 & 0,147 & $-0,0368$ \\
\hline NBI 2009 & 0,0549 & $-0,0423$ & $-0,1509$ & 0,083 & 0,0695 & 0,0634 & 0,0791 & $-0,0435$ \\
\hline VAM 2013 & 0,1512 & $-0,1732$ & $-0,104$ & $-0,0028$ & $-0,0192$ & 0,0333 & 0,0098 & $-0,0002$ \\
\hline IRCA 2013 & 0,1908 & $-0,1153$ & $-0,1513$ & $-0,0618$ & $-0,0192$ & 0,0024 & 0,084 & $-0,026$ \\
\hline
\end{tabular}

Nota: Infección Respiratoria Aguda (109), tumor maligno de estómago (201), enfermedades hipertensivas (302), enfermedades cerebrovasculares (307), accidentes de transporte terrestre (501), agresiones -homicidios- (512), diabetes mellitus (601), Mortalidad en la niñez (Mn).

una concentración de sus tasas acercándose a la diagonal de la igualdad.

En la mortalidad por tumor maligno de estómago, por hipertensión arterial, los homicidios y la Mortalidad en la niñez se presentan índices con valor negativo, indicando situación adversa en el comportamiento de la mortalidad para la población con mayores porcentajes de NBI. Situación contraria se observa en la mortalidad por Infección Respiratoria Aguda, por enfermedades isquémicas, por accidentes de tránsito y por diabetes mellitus.

La mortalidad por tumor maligno de estómago y por enfermedades hipertensivas evidenció un comportamiento más desfavorable para los municipios menos favorecidos al comparar por VAM. En cáncer gástrico, 30\% de los municipios con más bajo VAM acumulan $45 \%$ de las muertes, y en las enfermedades hipertensivas esta misma proporción de población aporta $40 \%$ de las muertes. En forma contraria, la mortalidad por Infección Respiratoria Aguda es más adversa en los municipios con mayor VMA (Tabla 7).

\section{Discusión}

La medición de desigualdades sociales constituye una herramienta fundamental para lograr la efectividad de un modelo de determinantes sociales. Es precisamente a partir de esta caracterización de las diferencias socioeconómicas de una sociedad y su relación con los diferentes niveles de salud que se hace posible un abordaje por determinantes. Este trabajo plantea ese esfuerzo para el departamento del Huila.

La mortalidad por IRA ha representado, a nivel nacional, cerca del 3,3\% del total de muertes en los últimos años, con tasas crudas entre 13 y 14 por 100.000 habitantes [10-12]. Para el Huila, aporta anualmente cerca del 3,1\% al total de muertes anuales, con tasas crudas entre 11,21 y 16,16 por 100.000 habitantes para los años 2010 y 2013, respectivamente. Las cifras nacional y departamental se ubican en valores inferiores frente al nivel mundial, donde anualmente se presentan cerca de 20 defunciones por 100.000 habitantes [13]. En las Américas, constituye una importante causa de muerte en menores de 10 años, principalmente en menores de 5 años y de 1 año; por el contrario, en los adolescentes y adultos es importante causa de morbilidad y de prestación de servicios [14]. El estudio de desigualdades en el ASIS Colombia 2002-2007 destaca, entre los factores de riesgo relacionados con IRA, determinantes ambientales como tipo de combustible para cocinar, estando asociado el riesgo a la leña, el carbón y la madera [7]. En nuestro estudio, se evidenció desigualdad en contra de los municipios con más ruralidad (clasificados como Eminentemente Rurales y Rurales) en la mortalidad por IRA, en consonancia con el riesgo ambiental descrito. También se destaca el estudio catalán de Pasarín, Borrel y Plasència [15], donde se advierte una diferencia notable en la mortalidad por neumonía en áreas con mayor desempleo en Barcelona.

El cáncer gástrico, para el presente estudio, representó al grupo de neoplasias. A nivel nacional, la mortalidad por esta causa es similar a la observada a nivel departamental, siendo la neoplasia de mayor mortalidad en la población general y en hombres, en estos últimos con tasas de 17,5 a 15,5 por 100.000 [16]. En las mujeres, se constituye en el tercer tipo de cáncer con mayor mortalidad, después del cáncer de mama y de tráquea, bronquios y pulmón, con tasas ajustadas entre 9,65 y 8,73 entre los años 2010 y 2014 [17]. El departamento se ubica en la segunda categoría más alta de tasas de mortalidad (11,71 a 14,90), junto con Tolima y otros siete departamentos, solo superados por Quindío, ubicado en solitario en la categoría de departamentos con tasas entre 14,91 y 22,30 por 100.000 habitantes, para el año 2011 [11].

Para el Huila, la mortalidad por cáncer gástrico, junto con las enfermedades hipertensivas, fue la causa que con mayor frecuencia evidenció un comportamiento adverso para los municipios menos favorecidos. Esta situación, en otras regiones del mundo, también se ha evidenciado. Un estudio del Reino Unido de 2005 evalúo la deprivación socioeconómica (mediante el Index of 


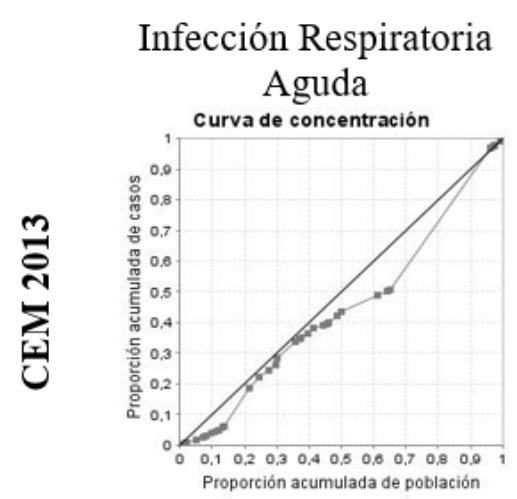

Índice de concentración:

0,1126
Tumor maligno de estómago

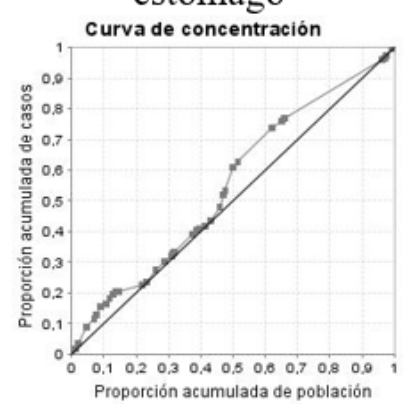

Índice de concentración: 0,0978

\section{Homicidio}

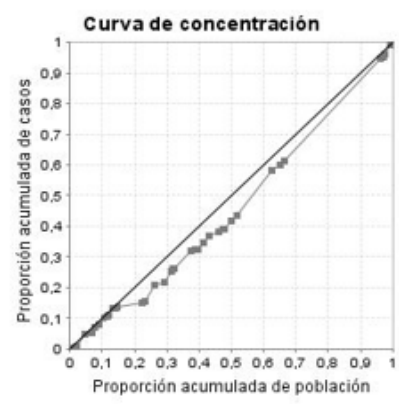

Índice de concentración: 0,0847

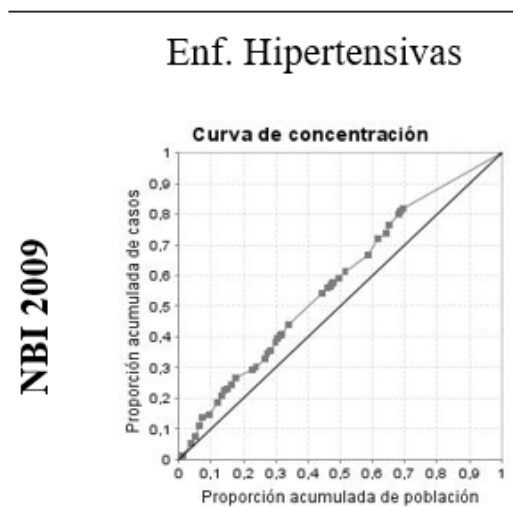

Índice de concentración:

0,1509

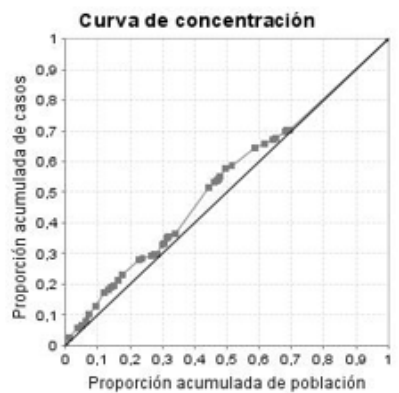

Índice de concentración:

0,0634
Diabetes mellitus

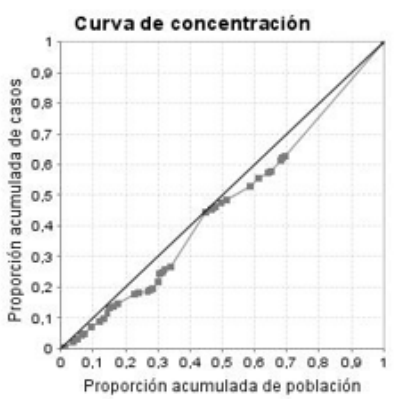

Índice de concentración:

0,0791

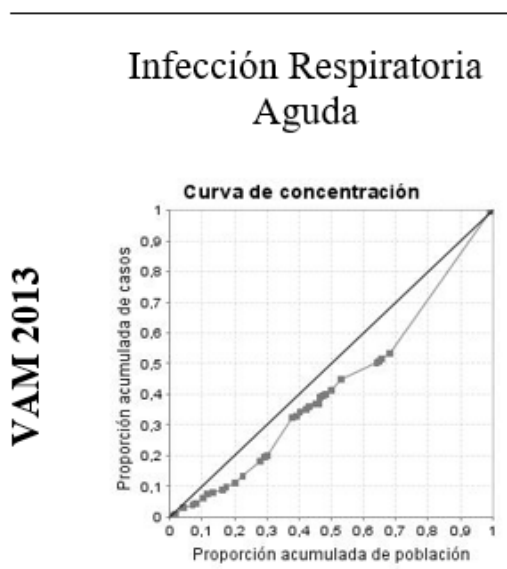

Índice de concentración:

0,1512

\section{Tumor maligno de estómago}

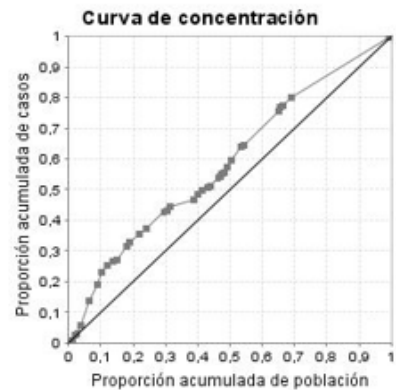

Índice de concentración: 0,1732
Enf. hipertensivas

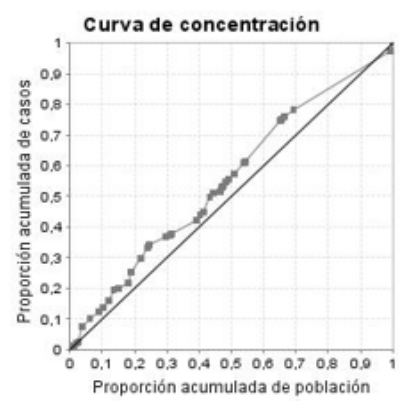

Índice de concentración: 0,104

Figura 2. Curvas de concentración según CEM 2013, NBI 2009 y VAM 2013 con mayor valor, en los municipios del Huila 2009 a 2013 
Multiple Deprivation -IMD-) frente a los resultados de pacientes con cáncer de estómago, identificando en las poblaciones menos favorecidas que la supervivencia a 5 años era del $32 \%$, frente al $66 \%$ de los más favorecidos y la mortalidad operatoria era tres veces mayor [18]. Adicionalmente, es reconocido a nivel mundial que la tasa de letalidad es más baja en países con más altos niveles de desarrollo humano [19].

Las tasas de mortalidad por enfermedades hipertensivas y cerebrovasculares en el departamento reflejan un mejor comportamiento frente a las cifras del país, donde, de acuerdo a los datos del ASIS 2015 [16], las tasas de mortalidad por enfermedades cerebrovasculares fueron entre 41,05 y 32,88 por 100.000 habitantes para el periodo 2005 a 2013, en las enfermedades hipertensivas las tasas oscilaron entre 15,48 y 16,51 por 100.000 habitantes, evidenciando un incremento en el periodo de observación. A su vez, la diabetes mellitus en el Huila presentó tasas ajustadas de mortalidad en promedio de 14,74 por 100.000 habitantes, con clara tendencia al descenso, pasando de 15,35 en 2009 a 13,25 para 2013, y con notable diferencia entre sexos, con una tasa en mujeres $76 \%$ superior a la tasa presentada en hombres. En Colombia, la tasa osciló entre 17,3 por 100.000 habitantes para el año 2010 y 15,3 para el año 2014 [17].

Para el Huila, las enfermedades hipertensivas evidenciaron un comportamiento adverso en todas las variables socioeconómicas evaluadas, para los municipios ubicados en el cuartil más desfavorecido, situación similar a lo observado en el estudio de Cardona y colaboradores en los municipios del eje cafetero, en donde enfrentan el PIB per cápita con el comportamiento de las mortalidades por eventos cardiovasculares, con notable diferencia en contra de los municipios más pobres [5]. De igual manera, la revisión de Fleischer y Diez [20] sobre las inequidades en enfermedades cardiovasculares en Latinoamérica señala la mayor tasa de mortalidad en zonas más vulnerables de Brasil, Chile y Argentina; en este último, principalmente asociados a la falta de empleo y el bajo nivel educativo.

Es importante señalar que el comportamiento adverso en la mortalidad por enfermedades hipertensivas para los municipios con mayor vulnerabilidad no se replica para las enfermedades cerebrovasculares, para las cuales, incluso en las variables de Cobertura en Educación, NBI y ruralidad, se observan mayores tasas en los municipios más favorecidos o menos vulnerables.

El Huila se incluye en el grupo de los cinco departamentos con mayores tasas de mortalidad por accidentes de tránsito, con tasas crudas y ajustadas en promedio de 20 por 100.000 habitantes durante el periodo 2009 a 2013, con una tendencia leve al descenso. En Colombia, las tasas crudas de mortalidad por accidente de tránsito han tenido una reducción de 14,68 muertes por 100.000 habitantes en 2009 , a 13,98 para el año 2013. La tasa mundial de mortalidad por accidentes de tránsito en 2013 se ubicó en 17,4 por 100.000 habitantes, 15,9 en las Américas, para Europa en 9,3 y en África, la región del mundo con mayor afectación, 26,6 por 100.000 habitantes [21]. Estos datos evidencian que las cifras departamentales de mortalidad por accidentes de tránsito superan la tendencia en Colombia, en las Américas y a nivel mundial.

En las mortalidades asociadas a la accidentalidad en tránsito, el estudio de Pasarín, Borrel y Plasència [15] señala el comportamiento adverso en las áreas básicas de salud con mayor desempleo. Para el Huila y sus municipios, la suficiencia económica presentó un comportamiento similar al estudio español, siendo esta variable socioeconómica la más cercana a la caracterización de empleo dentro de las variables del estudio. Por el contrario, en las otras variables el comportamiento fue muy disímil.

En homicidios, el Huila se ubica entre los departamentos con tasas inferiores a la media nacional. Para el año 2011, presentó una tasa cruda de homicidios de 25,51 por 100.000 habitantes. La violencia homicida en las Américas ocupa el primer lugar a nivel mundial, con una tasa promedio de 19,4 por 100.000 habitantes; se destacan Honduras, con 104 muertes por 100.000 habitantes, y Venezuela con 57,6, como los países con mayor violencia homicida. Colombia ocupa el sexto lugar, con una tasa de 43,9 por 100.000 habitantes, superado por El Salvador, Belice y Jamaica, además de Honduras y Venezuela [22]. A nivel nacional, se logró una extensa y muy completa caracterización de la violencia homicida en el $4^{\circ}$. Informe del ONS [23], en donde se destaca la diferenciación hombre/mujer, las altas tasas en la población de 15 a 44 años, la ausencia de desigualdades en el análisis según el Índice de Pobreza Multidimensional, es decir, no se estableció relación entre la pobreza municipal y las tasas de homicidio. En nuestro estudio, el NBI, indicador más cercano para la medición de pobreza, evidencia una leve tendencia a la mayor concentración de mortalidad en los municipios más pobres.

En la Mortalidad en la niñez, las cifras mundiales reportan, durante el periodo 2009 a 2013, una tasa media de 50 por 1.000 nacidos vivos, pasando de cerca de 12 millones de muertes en menores de 5 años, para el año 1990, a 7.6 millones en el año 2010, teniendo la mayor afectación los países del África Subsahariana y América Latina y el Caribe, más cerca de los países de ingresos altos que de los de ingresos bajos, con una tasa promedio de mortalidad en menores de 5 años de 20 por cada 1000 nacidos vivos [24]. Colombia 
ha tenido un comportamiento favorable y progresivo en los últimos años. De 1990 a 2010, el descenso en la mortalidad en menores de 5 años ha sido un poco mayor al 50\%, pasando de una tasa de 37 en 1990 a 19 en el año 2010 [25], y alcanzando 13,9 en el año 2013. El departamento del Huila se ubica entre los cinco departamentos con tasas de mortalidad en menores de 5 años más bajas a nivel nacional.

Para el año 2013, la tasa alcanzada fue de 12,4 por 1.000 nacidos vivos, un $10 \%$ inferior a la cifra nacional. El comportamiento no es homogéneo en el departamento, Colombia, Iquira y Paicol superan las 20 muertes por cada 1.000 nacidos vivos, resaltando al municipio de Iquira como uno de los que cuentan con mayor población indígena a nivel departamental. Ahora bien, la Mortalidad en la niñez, en nuestro estudio, junto con la mortalidad por cáncer gástrico y la mortalidad por enfermedades hipertensivas, es el evento en donde con mayor frecuencia se evidenciaron mayores tasas de mortalidad en los municipios con mayores vulnerabilidades socioeconómicas, situación similar a lo evidenciado a nivel mundial [24] [26] [27]. Los estudios coinciden en identificar, desde la perspectiva ecológica, relación entre el bajo ingreso y el bajo producto interno, con mayores tasas de mortalidad infantil y en la niñez. Según Schneider y colaboradores, $35 \%$ de las muertes de menores de 1 año se concentran en el $20 \%$ de los nacidos vivos, pertenecientes a los grupos con peor situación económica en América Latina [28].

El $6^{\circ}$ Informe del ONS sobre desigualdades en salud destaca, en la mortalidad en menores de 5 años, su desigual distribución en contra de la población con alta pobreza evaluada con el Índice de Pobreza Multidimensional y con base en la curva de concentración [8], hallazgo concordante con las desigualdades identificadas mediante el cruce con NBI en nuestro estudio.

\section{Conclusiones}

Entre todos los eventos de mortalidades observados, solo en la mortalidad por accidentalidad las cifras departamentales superan a la media nacional.

En la medición de desigualdades se identificaron tres eventos con constante desigualdad frente a las variables socioeconómicas evaluadas en contra de los municipios menos favorecidos: mortalidad por cáncer gástrico, enfermedades hipertensivas y Mortalidad en la niñez. Por el contrario, la mortalidad por infecciones respiratorias agudas y los homicidios, en todas las mediciones realizadas, evidenciaron un comportamiento menos adverso para los municipios más favorecidos. Estos resultados, en su conjunto, son poco comparables, dada la ausencia de estudios con un abordaje tan variado en cuanto a variables sanitarias y socioeconómicas confrontadas.
No se identificó una variable con constante comportamiento adverso frente a todas las mortalidades en estudio, situación que guarda sentido epidemiológico, considerando las diferencias de historia natural de la enfermedad para cada uno de los eventos.

\section{Referencias}

1 Organización Mundial de la Salud (OMS). Declaración política de Río sobre determinantes sociales de la salud [Internet]. Rio de Janeiro, Brasil; 2011 [Acceso 20 de noviembre de 2017]. Disponible en: http://www.who.int/sdhconference/declaration/ Rio_political_declaration_Spanish.pdf

2 Vega R. Informe Comisión Determinantes Sociales de la Salud de la Organización Mundial de la Salud. Rev Gerenc Polit Salud [revista en Internet] 2009 enero junio [Acceso 20 de noviembre de 2017]; 8(16):7-11. Disponible en: http://search.ebscohost.com/ login.asp $\mathrm{x}$.direct $=$ true $\& \mathrm{db}=\mathrm{a} 9 \mathrm{~h} \& \mathrm{AN}=47027039 \&$ lang $=$ es\&site $=$ ehost-live $\% 5$ Cnhttp://rev_gerenc_polit_salud.javeriana.edu.co/ vol8_n_16/editorial_vol8_n16.pdf

3 Comisión sobre Determinantes Sociales de la Salud (OMS). Subsanar las desigualdades en una generación. Alcanzar la equidad sanitaria actuando sobre los determinantes sociales de la salud [Internet]. Ginebra, Suiza: Global Health Promotion; 2008 [Acceso 20 de noviembre de 2017]. Disponible en: http://www. who.int/social_determinants/final_report/closethegap_how/es/

4 Whitehead M. Los conceptos y principios de la equidad en la salud [Internet]. Washington, D.C.: Oficina Sanitaria Panamericana, Oficina Regional de la OMS; 1991 [Acceso 20 de noviembre de 2017]. Disponible en: http://cursos.campusvirtualsp.org/ pluginfile.php/42712/mod resource/content/1/Whitehead - los conceptos y principios de la equidad en la salud OPS 1991.pdf

5 Cardona D, Gómez RD, Valecilla J, Cerezo M del P, Parra $\mathrm{JH}$, Cifuentes OL, et al. Desigualdades en la mortalidad por Enfermedades Cardiovasculares. Descripción de las desigualdades en los municipios del Eje Cafetero, 2009-2011. Duque Buitrago N, editor. Manizales, Colombia: Editorial Universidad Autónoma de Manizales; 2015. 226 p.

6 WHO (World Health Organization). Resolución WHA62.14. Reducir las inequidades sanitarias actuando sobre los determinantes sociales de la salud [Internet]. Ginebra, Suiza: 62a Asamblea Mundial de la Salud; 2009 [Accso 20 de noviembre de 2017]. Disponible en: http://apps.who.int/gb/ebwha/pdf files/ A62/A62_R14-sp.pdf

7 Ministerio de Salud y Protección Social. Análisis de la situación de salud en Colombia, 2002-2007. Tomo II Morbilidad y mortalidad de la población colombiana. Bogotá: Minsalud; 2012.

8 Observatorio Nacional de Salud Pública, INS. Desigualdades Sociales en salud en Colombia [Internet]. Bogotá: Imprenta Nacional de Colombia; 2015 [Acceso 20 de noviembre de 2017]. Disponible en: http://www.ins.gov.co/lineas-de-accion/ons/ SiteAssets/Paginas/publicaciones/6to informe ONS.pdf

9 Posada GJ. Agrupación de municipios colombianos según características de ruralidad. Medellín: Universidad Nacional de Colombia; 2010.

10 Ministerio de Salud y Protección Social. Indicadores básicos 2012 - Situación de salud en Colombia. Bogotá, Colombia: Ministerio de salud; 2013. p. 65.

11 Ministerio de Salud y la Protección Social. Indicadores Básicos 2013. Situación de Salud en Colombia [Internet]. Bogotá, Colombia: Minsalud; 2014 [Acceso 20 de noviembre de 2017]. 
Disponible en: https://www.minsalud.gov.co/sites/rid/Lists/ BibliotecaDigital/RIDE/VS/ED/PSP/Indicadores-basicossalud-2013.pdf

12 Ministerio de Salud y la Protección Social. Indicadores básicos 2014. Situación de Salud en Colombia [Internet]. Bogotá, Colombia: Minsalud; 2015 [Acceso 20 de noviembre de 2017]. Disponible en: https://www.minsalud.gov.co/sites/rid/Lists/ BibliotecaDigital/RIDE/VS/ED/PSP/Indicadores-basicossalud-2014.pdf

13 Ministerio de Salud y la Protección Social, Instituto Nacional de Salud. Protocolo de vigilancia en salud pública. Infección Respiratoria Aguda (IRA). [Internet]. Bogotá: Minsalud; 2016 [citado 10 de febrero 2015]. Disponible en: http://www.ins.gov.co/lineas-de-accion/SubdireccionVigilancia/sivigila/Protocolos\%20SIVIGILA/PRO\%20Infeccion\%20 Respiratoria\%20Aguda\%20IRA.pdf

14 Organización Panamericana de la Salud, OMS. Salud en las americas. Resumen: panorama regional y perfil de país. [Internet]. Washington: OPS; 2017. [Acceso 20 de noviembre de 2017]. Disponible en: http://www.paho.org/salud-en-las-americas-2017/ wp-content/uploads/2017/09/Print-Version-Spanish.pdf

15 Pasarín MI, Borrel C, Plasència A. ¿Dos patrones de desigualdades sociales en mortalidad en Barcelona? Gac Sanit [revista en Internet]. 1999 diciembre [Acceso 20 de noviembre de 2017]; 13(6) [431-440]. Disponible en: http://gacetasanitaria.org/es/vol13-num-6/sumario/S0213911199X70671/

16 Ministerio de Salud y la Protección Social. Análisis de Situación de Salud (ASIS), Colombia 2015 [Internet]. Bogotá, Colombia: Minsalud; 2015. [Acceso 20 de noviembre de 2017] Disponible en: https://www.minsalud.gov.co/sites/rid/Lists/BibliotecaDigital/ RIDE/VS/ED/PSP/asis-2015.pdf

17 Observatorio Nacional de Salud. Informe técnico. Carga de enfermedad por Enfermedades Crónicas No Transmisibles y Discapacidad en Colombia. Bogotá: Minsalud; 2015. p. 239.

18 Stephens MR, Blackshaw GR, Lewis WG, Edwards P, Barry JD, Hopper NA, et al. Influence of Socio-Economic Deprivation on Outcomes for Patients Diagnosed with Gastric Cancer. Scand J Gastroenterol 2005; 40(11): 1351-1357.

19 Stewart BW, Wild CP. World Cancer Report 2014. [Internet] Geneva: OMS, IARC; 2014 [Access 20 November 2017]. Available in: http://apps.who.int/bookorders/anglais/detart1.jsp? codlan $=1 \& \operatorname{cod} c o l=76 \& \operatorname{codcch}=31$

20 Fleischer NL, Diez AV. Inequidades en enfermedades cardiovasculares en Latinoamerica. Rev Peru Med Exp Salud Publica 2013; 30(4):641-648.

21 Organización Mundial de la Salud. Informe sobre la situación mundial de la seguridad vial 2015 [Internet]. Ginebra, Suiza: OMS; 2015. [Acceso 20 de noviembre de 2017]. Disponible en: http://www.who.int/violence_injury_prevention/road_safety_ status/2015/Summary_GSRRS2015_SPA.pdf

22 World Health Organization. World Health Statistics 2016: Monitoring Health for the Sdgs, Sustainable Development Goals [Internet]. Ginebra, Suiza: WHO; 2016. [Acceso 20 de nomviembre de 2017]. Available in: http://www.who.int/gho/ publications/world_health_statistics/EN_WHS08_Full.pdf

23 Ministerio de Salud y la Protección Social, Instituto Nacional de Salud, Observatorio Nacional de Salud (ONS).Violencia Homicida. Informe Técnico $4^{a}$ ed. Bogotá: Imprenta Nacional de Colombia; 2014.

24 Wagstaff A. Desigualdades socioeconómicas y mortalidad infantil: comparación de nueve países en desarrollo. Bull World Health Organ [revista en Internet]. 2000 [Acceso 20 de noviembre de 2017]; 78(1): [19-29]. Disponible en: internal-pdf://214.0.10.159/
Desigualdades Socioeconómicas ymortalidadinf. pdfLB-33070\%5Cnfile:///W:/PDFsen SALUD2008/ Wagstaff2000DesigualdadesSocioeconómicas.pdf

25 UNICEF, WHO, The World Bank, United Nations, DESA/ Population Division. Levels \& Trends in Child Mortality. Reporte 2011 [Internet]. New York, USA: United Nations Children's Fund; 2011. Available in: http://www.unicef.org/media/files/ Child_Mortality_Report_2011_Final.pdf

26 Pan American Health Organization (PAHO). Equity \& Health: Views from the Pan American Sanitary Bureau. Washington, D.C.: WHO; 2001. p.

27 Donoso E. Desigualdad en mortalidad infantil entre las comunas de la provincia de Santiago. Rev Med Chile 2004; (132): 461-466.

28 Schneider MC, Castillo-Salgado C, Bacallao J, Loyola E, Mujica OJ, Vidaurre M, et al. Métodos de medición de las desigualdades de salud. Rev Panam Salud Publica/Pan Am J Public Heal 2002; 12(6): 398-415.

29 Metzger X. La agregación de datos en la medición de desigualdades e inequidades en la salud de las poblaciones. Rev Panam Salud Publica/ Pan Am J Public Heal 2002; 12(6): 445-453. 